\title{
OPEN Toxoplasma gondii and multiple sclerosis: a population-based case-control study
}

\author{
Alessandra Nicoletti ${ }^{1,4} \bowtie$, Calogero Edoardo Cicero ${ }^{1,4}$, Loretta Giuliano ${ }^{1}$, Valeria Todaro ${ }^{1}$, \\ Salvatore Lo Fermo ${ }^{1}$, Clara Chisari ${ }^{1}$, Emanuele D'Amico ${ }^{1}$, Vincenza Paradisi ${ }^{2}$, \\ Antonia Mantella ${ }^{3}$, Alessandro Bartoloni ${ }^{3}$, Vito Sofia ${ }^{1}$, Francesco Patti ${ }^{1}$ \& Mario Zappia ${ }^{1}$
}

According to the hygiene hypothesis, parasites could have a protective role in the development of Multiple Sclerosis (MS). Our aim was to assess the association between presence of anti-Toxoplasma gondii antibodies and MS. MS patients were randomly selected from a population-based incident cohort of MS patients in the city of Catania. Age and sex-matched controls were randomly selected from the general population. Clinical and sociodemographic variables were recorded with a structured questionnaire and a blood sample was taken for serological analysis. Specific $T$. gondii IgG have been detected with a commercial kit. Adjusted Odds Ratios (ORs) were estimated using unconditional logistic regression. 129 MS subjects ( $66.7 \%$ women with a mean age $44.7 \pm 11.0$ years) and 287 controls (67.3\% women with a mean age $48.1 \pm 15.6$ years) have been enrolled in the study. Anti- $T$. gondii antibodies were found in 38 cases (29.5\%) and 130 controls (45.4\%) giving an adjusted OR of $0.56(95 \% \mathrm{Cl} 0.34-0.93)$. History of mononucleosis and high educational level were significantly associated with MS (adjOR 2.22 and 1.70 respectively) while an inverse association was found between high educational level and $T$. gondii seropositivity (adjOR 0.42 ). Our results further support the protective role of parasitic infections in MS.

Multiple Sclerosis (MS) is an inflammatory disease of the Central Nervous System (CNS) caused by an autoimmune process, characterized by a classical Th1 response ${ }^{1}$. MS is considered a multifactorial disease, due to a complex interaction between genetic and environmental factors. The impact of some environmental and lifestyle risk factors has been largely demonstrated, such as smoking, vitamin D levels and Epstein Barr virus (EBV) infection ${ }^{2}$. The role of infective agents is further supported by the "hygiene hypothesis", that postulates that infectious agents in an earlier age could act as a protective factor ${ }^{3}$. Hygiene hypothesis has been proposed as a possible explanation for the increased incidence of allergy and autoimmune diseases, including MS, in western countries and an imbalance between Th1 and Th2 cells was proposed as an immunological explanation ${ }^{4}$. As a matter of fact, epidemiological studies showed that prevalence of MS has been increasing over the time, especially in countries that have improved their socioeconomic and sanitation levels ${ }^{5}$ probably through a progressive decrease in the prevalence of infections ${ }^{6}$. Toxoplasma gondii (T. gondii) is an obligate intracellular protozoan replicating in the mammalian cells with a complex life cycle consisting of a sexual cycle in its feline definitive host and an asexual cycle in its intermediate host (livestock, birds, humans) ${ }^{7}$. It is estimated that at least one third of the general population is infected with T. gondii worldwide ${ }^{7}$. The risk of being infected with T. gondii is associated with a low socio-economic status ${ }^{8}$, the close contact with cats, and with eating raw or undercooked pork and lamb meat of infected hosts ${ }^{7}$. The clinical presentation of T. gondii infection is generally asymptomatic in immunocompetent humans, however in immunocompromised hosts it may give rise to more severe clinical manifestations, such as encephalitis ${ }^{7}$.

Several studies have suggested a possible association between chronic T. gondii infection and autoimmune diseases such as rheumatoid arthritis and systemic lupus erythematosus ${ }^{9}$, but the association between toxoplasmosis and MS is still unclear ${ }^{10}$.

\footnotetext{
${ }^{1}$ Department of Medical and Surgical Sciences and Advanced Technologies "G.F. Ingrassia", Section of Neurosciences, University of Catania, Via Santa Sofia 78, 95123 Catania, Italy. ${ }^{2}$ Italian Society of General Medicine (SIMG), Catania, Italy. ${ }^{3}$ Department of Experimental and Clinical Medicine, Infectious and Tropical Diseases Unit, University of Florence, Florence, Italy. ${ }^{4}$ These authors contributed equally: Alessandra Nicoletti and Calogero Edoardo Cicero. ${ }^{\boxplus}$ email: anicolet@unict.it
} 
In fact, a recently published metanalysis of studies assessing the association between MS and T. gondii has demonstrated a lower, but still not significant, protective effect of the parasite on the risk of being diagnosed with $\mathrm{MS}^{10}$. However, all of the studies included in the meta-analysis have relatively small sample sizes ${ }^{10}$, negatively impacting the statistical power of the studies. In order to overcome these limitations, we carried out a populationbased case-control study to better evaluate the relationship between T. gondii and MS.

\section{Materials and methods}

Study population. This study is part of a population-based case-control study conducted by our group in the city of Catania to assess the association between environmental risk factors and MS. The study gathered clinical data and serum samples from 164 MS patients and 481 age and sex matched controls. Background and methods have been extensively reported elsewhere ${ }^{11}$. Briefly, MS patients were randomly selected from an incident cohort of MS patients consisting of all incident cases in the city of Catania who had had the onset of disease from 1st January 1975 to 31 st December 2004 and comprises 367 MS patients ${ }^{12-14}$. From this cohort 164 MS patients, diagnosed according to Poser's criteria ${ }^{15}$ were randomly selected.

Population controls were enrolled in the study through an "equal probability selection method" (EPSEM). At least three control subjects per each MS patient, frequency matched by age and sex were enrolled from the general population of Catania using multistage sampling methods.

Control subjects, not affected by neurological disorders, were randomly selected from General Practitioners' cabinet list.

Blood sample collection and analysis. At the time of the enrolment $20 \mathrm{cc}$ of whole blood were taken for each subject. Serum samples were analysed at the laboratories of the Infectious Diseases Institute of the University of Florence and have been analysed by a biologist blinded to the status of the participants. Specific Toxoplasma gondii IgG have been detected with a commercial ELISA kit (DIA.PRO Toxoplasma IgG; Diagnostic Bioprobes s.r.l.) with a sensitivity and specificity reported by the manufacturer of $98 \%$.

Assessment of exposures. A face-to-face semi-structured standardized questionnaire investigating environmental exposures and comorbidities has been administered to all patients and controls. All subjects have been asked the questions directly and in the case of a disability-related impairment in communication, to a proxy responder, preferably the spouse or sibling. Details of the screening instrument are reported elsewhere ${ }^{11}$.

Statistical analysis. A double entry process was implemented to enter all the gathered clinical, laboratory and demographic variables into the dataset. Data cleaning included range, consistency checks and cross referencing with original data to identify and correct missing values. To describe the variables, frequency, means and standard deviations were used. Variables normally distributed after the Shapiro-Wilk test were analysed with the two tailed t test. Otherwise a Mann-Whitney test was conducted. For qualitative variables significance testing we employed the chi squared and Fisher test. For the prevalence of anti-T. gondii antibodies the 95\% CI have been calculated. Statistical significance was set at $\mathrm{p}<0.05$. In order to test the association between positivity to T. gondii antibodies and explanatory variables we conducted an unconditional logistic regression analysis (two-tailed, $\alpha=0.05$ ). Variables associated with a $\mathrm{p} \leq 0.25$ were then used to compute the multivariate model. We manually compared the log-likelihood of the model with and without a specific variable with the likelihood ratio test. Sex and age have been considered a priori confounder variables.

The study has been performed in accordance with STROBE (Strengthening The Reporting of Observational Studies in Epidemiology) guidelines (Supplementary methods 1). All the analyses have been conducted with the software STATA 12.0 .

Ethics. All the patients and controls enrolled in the study have been given a paper briefly explaining the reason of the study and have been resumed orally by the GP or the neurologist. A written informed consent model has then been signed by the patient or the control or a legal representative in the case of severe disability if they accepted to participate in the study. The study has been approved by the Ethical Committee of the AOU Policlinico Vittorio Emanuele with the code 64/2018/PO.

\section{Results}

The original sample of the case-control study consisted of 164 cases and 481 controls. Baseline characteristics (sex, age at onset, disease form and expanded disability status scale) of the 164 enrolled patients were not significantly different from the whole MS population (incident cohort of MS patients consisting of 367 patients) ${ }^{11}$. Out of the 164 MS patents a serum sample was available for 129 (78.6\%) cases and 287 (59.6\%) controls.

At the time of the enrolment, MS patients had a mean age of $44.7 \pm 11.0$ years, and $86(66.7 \%)$ were women; mean age of control subjects was $48.1 \pm 15.6$ while 193 were women (67.3\%). Mean age at onset was $32.7 \pm 11.3$ years with a mean disease duration of $12.0 \pm 8.2$ years. The most frequent type of MS was the RR form $(\mathrm{n}=103 ; 79.8 \%)$ and the mean expanded disability status scale (EDSS) at the enrolment was $2.5 \pm 2.2$. The majority of patients was under treatment with an immunomodulating drug $(n=76 ; 59.8 \%)$ while only $17(13.4 \%)$ were not taking any therapy, as reported in Table 1.

Presence of anti-T. gondii antibodies was detected in 38 patients giving a seroprevalence of $29.5 \%$ (95\%CI $21.8-38.1)$ and 130 controls resulting in a seroprevalence of $45.4 \%$ (95\%CI 39.6-51.4) giving an OR of 0.50 $(95 \%$ CI $0.32-0.78 ;$-value $=0.002)$. 


\begin{tabular}{|c|c|}
\hline & Multiple Sclerosis patients $(n=129)$ \\
\hline Age, $y($ mean \pm SD $)$ & $44.7 \pm 11.0$ \\
\hline Sex (women), n (\%) & $86(66.7)$ \\
\hline $\begin{array}{l}\text { Age at onset, y (mean } \\
\pm \text { SD) }\end{array}$ & $32.7 \pm 11.3$ \\
\hline $\begin{array}{l}\text { Disease duration, } y \\
(\text { mean } \pm \text { SD) }\end{array}$ & $12.0 \pm 8.2$ \\
\hline \multicolumn{2}{|l|}{ Disease type, n (\%) } \\
\hline Relapsing Remitting & $103(79.8)$ \\
\hline Secondary Progressive & $23(17.0)$ \\
\hline Primary Progressive & $2(1.5)$ \\
\hline Other & $2(1.5)$ \\
\hline EDSS, mean \pm SD & $2.5 \pm 2.2$ \\
\hline \multicolumn{2}{|l|}{ Treatment, n (\%) } \\
\hline No therapy & $17(13.4)$ \\
\hline Immunomodulators & $76(59.8)$ \\
\hline Immunosuppressants & $20(15.7)$ \\
\hline Monoclonal antibodies & $14(11.0)$ \\
\hline $\begin{array}{l}\text { T. gondii seropositivity, } \\
\text { n (\%) }\end{array}$ & $38(29.5)$ \\
\hline
\end{tabular}

Table 1. Demographic characteristics of Multiple Sclerosis patients. n, number; SD, standard deviation; EDSS, expanded disability status scale.

We have also previously assessed the presence of antibodies anti Toxocara canis (T. canis $)^{16}$. Eleven MS cases (8.5\%) and $23(8.0 \%)$ controls presented antibodies anti T. canis. Four MS cases (3.1\%) and 14 (4.9\%) controls presented a dual infection and were seropositive for both $T$. canis and T. gondii. Presence of dual infection was not significantly associated with MS at univariate analysis (OR $0.62 ; 0.20-1.93 ;$-value 0.4 ).

At univariate analysis MS was strongly associated with education with a significant trend (chi square 23.5; p-value 0.0003 ) reaching a strongest positive association for university degree (OR 6.87; 95\%CI 2.90-16.20; p-value $<0.0001)$; MS was also positively associated with history of mononucleosis and inversely associated with history of autoimmune diseases as reported in Table 2.

At the multivariate analysis, after adjusting for age and sex considered as a priori confounders, a significant negative association was found between MS and T. gondii seropositivity (adjOR 0.56; 0.34-0.93; p-value 0.02) and history of autoimmune diseases (adjOR $0.32 ; 0.12-0.81 ; p$-value 0.02 ), while a positive association was found with years of schooling (adjOR 1.70; 1.07-2.73; p-value 0.02) and history of mononucleosis (adjOR 2.22; $1.12-4.39 ; p$-value 0.02$)$. Forcing occupation in the final model did not modify the strength of the association with T. gondii (adjOR 0.56; 0.32-0.97; p-value 0.04).

Both at univariate and multivariate analysis (adjusting by age and sex respectively) T. gondii sero-positivity was significantly associated with age (adjOR 1.06; 95\%CI 1.05-1.08; p-value $<0.0001$ ) and female sex (1.97; 95\%CI 1.04-1.08; p-value $<0.0001$ ), but not with the presence of cats or other domestic animals (dogs, birds). Furthermore, a strong negative association was found between education and T. gondii seropositivity (years of schooling $\geq 9$ years) with an adjOR of 0.42 (95\%CI $0.27-0.65$; $p$-value $<0.0001)$.

Considering only the control population 33 subjects (12.3\%) out of 269 reported a positive history for autoimmune diseases other than MS (25 autoimmune thyroiditis, seven rheumatoid arthritis and one LES). We found a negative association, even if borderline significant, between $T$. gondii seropositivity and presence of autoimmune diseases $(0.54$; $95 \% \mathrm{CI} 0.25-1.16$; p-value 0.1$)$. This association was stronger and significant when adjusting by age and sex (adj OR 0.38; 95\%CI 0.16-0.90; p-value 0.03) (Supplementary Table S1).

\section{Discussion}

We found a negative association between T. gondii and MS, suggesting a possible protective role of this parasite and supporting the hygiene hypothesis. According to the hygiene hypothesis, in fact, low exposure to pathogens early in life can increase the risk for autoimmune diseases, including MS and allergy, especially in western countries probably due to an immunological imbalance between Th1 and Th2 response ${ }^{4}$.

However, parasites are divided into two major groups, helminths and protozoans, that can act with different immunological mechanisms. Helminths, in fact, induce a Th2 response characterized by the production of IL-4, IL-5, and IL- $10^{17}$ while protozoans induce a very strong Th1 response by the production of pro-inflammatory mediators, such as IL-12, IFN- $\gamma$, and nitric oxide ${ }^{18}$.

Helminths modulation of autoimmune response has been shown in different autoimmune diseases ${ }^{19-21}$. In MS, the protective influence of helminths is mediated by the down modulation of inflammatory responses and enhancement of immune regulation ${ }^{22}$. Some experimental and epidemiological evidences have supported this hypothesis. In particular, infection with Schistosoma mansoni has demonstrated to exert a protective effect on the development of Experimental Allergic Encephalomyelitis (EAE) in infected mice reducing IFN- $\gamma$, tumor 


\begin{tabular}{|c|c|c|c|c|c|c|c|c|}
\hline & \multirow{2}{*}{$\begin{array}{l}\text { Multiple Sclerosis } \\
\text { patients } \\
(\mathrm{n}=129)\end{array}$} & \multirow{2}{*}{$\begin{array}{l}\text { Controls } \\
(\mathbf{n}=287)\end{array}$} & \multicolumn{3}{|c|}{ Univariate analysis } & \multicolumn{3}{|c|}{ Multivariate analysis } \\
\hline & & & OR & 95\% CI & p-value & adjOR & $95 \% \mathrm{CI}$ & p-value \\
\hline Age, y (mean \pm SD) & $44.7 \pm 11.0$ & $48.1 \pm 15.6$ & 0.98 & $0.97-1.00$ & 0.03 & 0.99 & $0.98-1.01$ & 0.6 \\
\hline Sex (women), n (\%) & $86(66.7)$ & $193(67.3)$ & 0.97 & $0.62-1.51$ & 0.9 & 1.07 & $0.66-1.72$ & 0.8 \\
\hline \multicolumn{9}{|l|}{ Profession, n (\%) } \\
\hline Professional/Office job & $51(46.5)$ & $90(31.8)$ & 1 & 1 & 1 & & & \\
\hline Industry & $6(5.4)$ & $39(13.8)$ & 0.27 & $0.11-0.68$ & 0.006 & & & \\
\hline Tradesman & $4(3.6)$ & $9(3.2)$ & 0.78 & $0.23-2.67$ & 0.7 & & & \\
\hline Artisan & $3(2.7)$ & $7(2.5)$ & 0.75 & $0.18-3.05$ & 0.7 & & & \\
\hline Agricolture & 1 & $1(0.3)$ & 1 & 1 & 1 & & & \\
\hline Housewife & $25(22.7)$ & 79 (27.9) & 0.56 & $0.32-0.98$ & 0.04 & & & \\
\hline Unemployed & $9(8.1)$ & $10(3.5)$ & 1.58 & $0.61-4.16$ & 0.5 & & & \\
\hline Other & $12(10.9)$ & $48(17.0)$ & 0.44 & $0.21-0.97$ & 0.03 & & & \\
\hline $\begin{array}{l}\text { Number of siblings (mean } \\
\pm \text { SD) }\end{array}$ & $2.97 \pm 2.0$ & $3.5 \pm 2.9$ & 0.92 & $0.83-1.001$ & 0.08 & & & \\
\hline \multicolumn{9}{|l|}{ Education, n (\%) } \\
\hline Illiterate/primary school & $8(6.2)$ & $66(23.0)$ & 1 & 1 & 1 & & & \\
\hline Secondary school & 38 (29.5) & $94(32.7)$ & 3.33 & $1.46-7.60$ & 0.004 & & & \\
\hline High school & $48(37.2)$ & 85 (29.6) & 4.66 & $2.06-10.5$ & $<0.0001$ & & & \\
\hline University & $35(27.1)$ & $42(14.6)$ & 6.87 & $2.90-16.25$ & $<0.0001$ & & & \\
\hline \multicolumn{9}{|l|}{ Years of schooling, $n$ (\%) } \\
\hline$\leq 8$ years & $46(35.7)$ & $160(55.7)$ & 1 & 1 & 1 & 1 & 1 & 1 \\
\hline$\geq 9$ years & $83(64.3)$ & $127(44.2)$ & 2.27 & $1.48-3.49$ & $<0.0001$ & 1.70 & $1.07-2.73$ & 0.02 \\
\hline Cat ownership, n (\%) & $41(31.8)$ & $92(32.1)$ & 0.99 & $0.63-1.54$ & 0.9 & & & \\
\hline Dog ownership, n (\%) & $63(48.8)$ & $135(47.0)$ & 1.07 & $0.71-1.63$ & 0.7 & & & \\
\hline T. gondii positivity, n (\%) & 38 (29.5) & $130(45.4)$ & 0.50 & $0.32-0.78$ & 0.002 & 0.56 & $0.34-0.93$ & 0.02 \\
\hline T. canis positivity, n (\%) & $11(8.5)$ & $23(8.0)$ & 1.07 & $0.50-2.27$ & 0.2 & & & \\
\hline $\begin{array}{l}\text { T. gondii and T. canis } \\
\text { positivity, } \mathrm{n}(\%)\end{array}$ & $4(3.1)$ & $14(4.9)$ & 0.62 & $0.20-1.93$ & 0.4 & & & \\
\hline Mononucleosis, n (\%) & $22(17.0)$ & $20(7.1)$ & 2.70 & $1.42-5.16$ & 0.003 & 2.22 & $1.12-4.39$ & 0.02 \\
\hline $\begin{array}{l}\text { Autoimmune diseases, } \\
\text { n (\%) }\end{array}$ & $6 / 129(4.6)$ & $33 / 269(12.3)$ & 0.34 & $0.14-0.85$ & 0.02 & 0.32 & $0.12-0.81$ & 0.02 \\
\hline $\begin{array}{l}\text { Smoking (Never/Ever), } \\
\text { n (\%) }\end{array}$ & $56 / 107(52.3)$ & $127(44.4)$ & 1.11 & $0.80-1.53$ & 0.5 & & & \\
\hline
\end{tabular}

Table 2. Univariate and multivariate analysis. Significant $\mathrm{p}$ values are highlighted in bold. n, number; SD, standard deviation; OR, odds ratio; CI, confidence intervals; adjOR, Adjusted OR.

necrosis factor alpha (TNF- $\alpha$ ) and IL-12 production and dampening the classical Th1 response ${ }^{23}$. These effects are thought to be associated with the immunological switch to a Th2 response caused by parasite infestation ${ }^{24}$.

Apart from helminths, recently interest has been drawn to $T$. gondii, an intracellular parasite that causes toxoplasmosis in both humans and animals. Several studies have suggested an association between chronic $T$. gondii infection and autoimmune diseases such as diabetes mellitus type I, rheumatoid arthritis and systemic lupus erythematosus, but conflicting results have been reported, suggesting that $T$. gondii may play a positive or negative role on different autoimmune conditions ${ }^{9,25,26}$.

In the last years some studies have also investigated the association between toxoplasmosis and MS. To the best of our knowledge up to date only five hospital-based case-control studies ${ }^{26-30}$ have been carried out and all, except one ${ }^{28}$, reported a negative association, even if it was significant only in two studies ${ }^{27,29}$. Nonetheless it should be noted that for the majority of these studies sample size was very small, thus the lack of significance may be also attributable to a lack of adequate power. Only one $s t u d y^{28}$ reported a positive, but not significant, association between T. gondii infection and MS, but also in this case sample size was particularly small (52 MS patients and 45 controls $)^{28}$. All these previously conducted studies have been included in a recent metanalysis (669 MS patients and 770 controls) ${ }^{10}$ where a lower pooled seroprevalence of $T$. gondii was recorded among MS patients (32.4\% versus $39.1 \%$ ) leading to a negative, even if not significant, association with a combined OR of 0.72 (95\% CI: $0.49-1.06)^{10}$.

Our study represents one of the largest case-control studies aimed to assess the possible association between T. gondii and MS and the only one with a population-based design, avoiding possible selection bias. On average, seroprevalence detected among cases $(29.5 \%)$ and controls $(45.4 \%)$ was close to those reported in literature ${ }^{10}$ with a significant negative association between T. gondii seropositivity and MS with an OR of 0.50 .

It should be underlined that, in agreement with literature data, history of mononucleosis was positively associated with $\mathrm{MS}^{22}$. On the other hand, along with $T$. gondii seropositivity, in our sample also positive history for other autoimmune diseases was negatively associated with MS suggesting a possible protective effect. Even 
if some studies suggest an increased frequency of some autoimmune disease among MS patients the possible relationship between MS and other autoimmune diseases is still debated ${ }^{31}$. However, it should be noted that the presence of autoimmune disease in our study was based just on clinical history, thus this finding should be interpreted with caution.

Considering the T. gondii seropositivity, in agreement with literature, age and lower educational level represented a possible risk factor ${ }^{8,32}$, while we did not find any significant association between pet ownership and $T$. gondii seropositivity. Furthermore, MS was associated with higher levels of education with a significant trend, reaching an OR of 6.87 for those who attended the university ${ }^{33,34}$. Conversely higher levels of education were associated with lower prevalence of $T$. gondii antibodies. Education is a proxy of the socioeconomic status that is generally associated with increased levels of sanitation, thus with a reduced exposition to different pathogens during childhood. This evidence is also in agreement with the hygiene hypothesis, as highlighted by some initial MS studies performed in the $90 \mathrm{~s}^{33,34}$.

Our findings provide a further support for the hygiene hypothesis. However, as for other protozoans, it should be noted that T. gondii induces a very strong Th1 response rather than a characteristic Th2 response observed for helminths ${ }^{35}$. As a matter of fact literature data suggest that a possible protective role of $T$. gondii could be exerted through an increased production of IL- $10^{35}$. Indeed, studies using both human and animal models have suggested a number of different underlying mechanisms. In particular in animal models it has been demonstrated that $T$. gondii not only induces the production of IFN- $\gamma$ in T-bet + T helper cells, but also of IL-10, indicating that the parasite elicits regulatory activities in its acute and chronic phase $\mathrm{e}^{9,35}$. According to literature IL-10 could play a relevant role in reducing autoimmune reaction in $\mathrm{MS}^{36}$, thus, as for helminth models, it is possible to hypothesize that $T$. gondii could exert a protective effect through the increase of IL-10 levels.

Moreover, host response to toxoplasmosis acute infection is characterized by a strong inflammatory activation mediated by the Th17 CD4 lymphocytes producing IL-1737. In turn, T. gondii has developed survival mechanisms that allow for the parasite survival through the dampening of the Th-17 response via the induction of IL-27 production by antigen presenting cells ${ }^{37}$. Interestingly, high levels of IL-27 have been demonstrated in cerebrospinal fluid of MS patients probably exerting an antinflammatory activity ${ }^{38}$.

Another possible explanation of the immunosuppressive activity of T. gondii infection can be related to the activation of the FoxP3 + T cells, which dampen the immune system response during chronic T. gondii infection probably by reducing the availability of IL-2, a strong proinflammatory cytokine ${ }^{39}$. Lastly, also TGF-beta is relevant in the immunosuppression present during the chronic T. gondii infection ${ }^{40}$, a cytokine that plays a major role in the promotion of CD4 regulatory activity in MS patients ${ }^{41}$.

Nonetheless, to date, no data are available on experimental infection of T. gondii in EAE mice, the mouse model of MS, thus studies are needed to understand the underlying pathophysiological mechanisms.

In the attempt to understand the possible underlining mechanisms that could explain the relationship between parasitic infections and autoimmune diseases, in a previous study involving the same sample of cases and controls we evaluated the association between MS and T. canis, one of the most prevalent helminthiases worldwide, but we did not find any significant association ${ }^{16}$.

From a methodological point of view our studies presents many strengths. This is the first population-based case-control study carried out to evaluate the possible association between MS and T. gondii. MS patients, in fact, were randomly enrolled from a well-established cohort of incident MS patients, while controls were selected from the general population using a multistage sampling method ${ }^{11}$, thus reducing the risk of a possible selection bias among cases. Furthermore, along with the study carried out by Stascheit et coll. in $2015^{27}$, our study represents the largest case-control study carried out to investigate the possible relationship between MS and T. gondii.

Along with selection bias, recall bias represents the second most common type of bias affecting case-control studies. However, in our study the possible role of recall bias can be ruled out since exposition to T. gondii has been assessed by the detection of serum IgG antibodies and the biologist who performed the serological assessment was blinded with respect to the disease status.

Nonetheless the retrospective nature of the case-control design largely limits the interpretation of our results. We cannot exclude the role of a low socioeconomic status in modulating the interaction between T. gondii infection and MS considering that it acts as a risk factor for T. gondii and as protective factor for MS. However, we have conducted a multivariate model including the level of education and occupational status considered both proxies of socioeconomic status and we found a still significant protective effect of T. gondii on MS. Moreover, the role of eating raw/undercooked meat and its relation to higher risk of $T$. gondii, was not explored due to the lack of this information on the original case-control study. At any rate, while there is an increased risk of infection with T. gondii for consuming raw/undercooked meat ${ }^{7}$, the association between meat consumption and MS is still unclear ${ }^{42}$.

Indeed we are unable to establish the exact sequence of the events, thus we cannot exclude that the exposition to T. gondii has been occurred after the disease onset. Nonetheless it should be underlined that a previous study found no association between disease duration and antibodies load ${ }^{29}$.

In conclusion even if our study supports the possible protective role of T. gondii on MS, further clinical and experimental studies are needed to confirm our results and to understand the underlying pathophysiological mechanisms ${ }^{27}$.

\section{Data availability}

The datasets generated during and/or analysed during the current study are available from the corresponding author on reasonable request. 
Received: 4 April 2020; Accepted: 9 October 2020

Published online: 02 November 2020

\section{References}

1. Hemmer, B., Kerschensteiner, M. \& Korn, T. Role of the innate and adaptive immune responses in the course of multiple sclerosis. Lancet Neurol. 14, 406-419 (2015).

2. Olsson, T., Barcellos, L. F. \& Alfredsson, L. Interactions between genetic, lifestyle and environmental risk factors for multiple sclerosis. Nat. Rev. Neurol. 13, 25-36 (2017).

3. Correale, J., Farez, M. F. \& Gaitán, M. I. Environmental factors influencing multiple sclerosis in Latin America. Mult. Scler. J. Exp. Transl. Clin. 3, 2055217317715049 (2017).

4. Stiemsma, L. T., Reynolds, L. A., Turvey, S. E. \& Finlay, B. B. The hygiene hypothesis: current perspectives and future therapies. Immunotargets Ther. 4, 143-157 (2015).

5. Cabre, P. et al. Role of return migration in the emergence of multiple sclerosis in the French West Indies. Brain 128, 2899-2910 (2005).

6. Fleming, J. O. \& Cook, T. D. Multiple sclerosis and the hygiene hypothesis. Neurology 67, 2085-2086 (2006).

7. Tenter, A. M., Heckeroth, A. R. \& Weiss, L. M. Toxoplasma gondii: from animals to humans. Int. J. Parasitol. 30, 1217-1258 (2000).

8. Mareze, M. et al. Socioeconomic vulnerability associated to Toxoplasma gondii exposure in southern Brazil. PLoS ONE 14, e0212375 (2019).

9. Fischer, S. et al. Toxoplasma gondii: bystander or cofactor in rheumatoid arthritis. Immunol. Res. 56, 287-292 (2013).

10. Saberi, R. et al. Is Toxoplasma gondii playing a positive role in multiple sclerosis risk? A systematic review and meta-analysis. J. Neuroimmunol. 322, 57-62 (2018).

11. Nicoletti, A. et al. Risk factors in multiple sclerosis: a population-based case-control study in Sicily. Background and methods. Neurol. Sci. 37, 1931-1937 (2016).

12. Nicoletti, A. et al. Prevalence and incidence of multiple sclerosis in Catania, Sicily. Neurology 56, 62-66 (2001).

13. Nicoletti, A. et al. Possible increasing risk of multiple sclerosis in Catania, Sicily. Neurology 65, 1259-1263 (2005).

14. Nicoletti, A. et al. Increasing frequency of multiple sclerosis in Catania, Sicily: a 30-year survey. Mult. Scler. 17, 273-280 (2011).

15. Poser, C. M. et al. New diagnostic criteria for multiple sclerosis: guidelines for research protocols. Ann. Neurol. 13, 227-231 (1983).

16. Cicero, C. E. et al. Lack of association between Toxocara canis and multiple sclerosis: a population-based case-control study. Mult. Scler. 26, 258-259 (2020).

17. Jackson, J. A., Friberg, I. M., Little, S. \& Bradley, J. E. Review series on helminths, immune modulation and the hygiene hypothesis: immunity against helminths and immunological phenomena in modern human populations: coevolutionary legacies?. Immunology 126, 18-27 (2009).

18. Munoz, M., Liesenfeld, O. \& Heimesaat, M. M. Immunology of Toxoplasma gondii. Immunol. Rev. 240, 269-285 (2011).

19. Zaccone, P. \& Hall, S. W. Helminth infection and type 1 diabetes. Rev. Diabet. Stud. 9, 272-286 (2012).

20. Elliott, D. E. \& Weinstock, J. V. Helminth-host immunological interactions: prevention and control of immune-mediated diseases. Ann. N. Y. Acad. Sci. 1247, 83-96 (2012).

21. Versini, M. et al. Unraveling the Hygiene Hypothesis of helminthes and autoimmunity: origins, pathophysiology, and clinical applications. BMC Med. 13, 81 (2015).

22. Correale, J. \& Gaitán, M. I. Multiple sclerosis and environmental factors: the role of vitamin D, parasites, and Epstein-Barr virus infection. Acta Neurol. Scand. 132, 46-55 (2015).

23. La Flamme, A. C., Ruddenklau, K. \& Bäckström, B. T. Schistosomiasis decreases central nervous system inflammation and alters the progression of experimental autoimmune encephalomyelitis. Infect. Immun. 71, 4996-5004 (2003).

24. Sewell, D. et al. Immunomodulation of experimental autoimmune encephalomyelitis by helminth ova immunization. Int. Immunol. 15, 59-69 (2003).

25. Krause, I. et al. Anti-infectious antibodies and autoimmune-associated autoantibodies in patients with type I diabetes mellitus and their close family members. Ann. N. Y. Acad. Sci. 1173, 633-639 (2009).

26. Shapira, Y. et al. Prevalence of anti-Toxoplasma antibodies in patients with autoimmune diseases. J. Autoimmun. 39, 112-116 (2012).

27. Stascheit, F., Paul, F., Harms, L. \& Rosche, B. Toxoplasma gondii seropositivity is negatively associated with multiple sclerosis. J. Neuroimmunol. 285, 119-124 (2015).

28. Oruç, S. et al. Relationship of Toxoplasma gondii exposure with multiple sclerosis. Eur J. Gen. Med. 13, 58-63 (2016).

29. Koskderelioglu, A., Afsar, I., Pektas, B. \& Gedizlioglu, M. Is Toxoplasma gondii infection protective against multiple sclerosis risk?. Mult. Scler. Relat. Disord. 15, 7-10 (2017).

30. Pestehchian, N. et al. Frequency of blood-tissue parasitic infections in patients with multiple sclerosis, as compared to their family members. Int. J. Prev. Med. 5, 1578-1581 (2014).

31. Marrie, R. A. et al. A systematic review of the incidence and prevalence of autoimmune disease in multiple sclerosis. Mult. Scler. 21, 282-293 (2015).

32. Wang, T. et al. Seroprevalence of Toxoplasma gondii infection in blood donors in mainland China: a systematic review and metaanalysis. Parasite 25, 36 (2018).

33. Kurtzke, J. F. \& Page, W. F. Epidemiology of multiple sclerosis in US veterans: VII. Risk factors for MS. Neurology 48, 204-213 (1997).

34. Hammond, S. R., McLeod, J. G., Macaskill, P. \& English, D. R. Multiple sclerosis in Australia: socioeconomic factors. J. Neurol. Neurosurg. Psychiatry 61, 311-313 (1996).

35. Jankovic, D. et al. Conventional T-bet(+)Foxp3(-) Th1 cells are the major source of host-protective regulatory IL-10 during intracellular protozoan infection. J. Exp. Med. 204, 273-283 (2007).

36. Correale, J., Farez, M. \& Razzitte, G. Helminth infections associated with multiple sclerosis induce regulatory B cells. Ann. Neurol. 64, 187-199 (2008).

37. Stumhofer, J. S. et al. Interleukin 27 negatively regulates the development of interleukin 17 -producing T helper cells during chronic inflammation of the central nervous system. Nat. Immunol. 7, 937-945 (2006).

38. Lalive, P. H. et al. Increased interleukin-27 cytokine expression in the central nervous system of multiple sclerosis patients. $J$. Neuroinflam. 14, 144 (2017).

39. Tenorio, E. P., Fernández, J., Castellanos, C., Olguín, J. E. \& Saavedra, R. CD4+ Foxp3+ regulatory T cells mediate Toxoplasma gondii-induced T-cell suppression through an IL-2-related mechanism but independently of IL-10. Eur. J. Immunol. 41, 3529-3541 (2011).

40. Zare-Bidaki, M. et al. TGF- $\beta$ in toxoplasmosis: friend or foe?. Cytokine 86, 29-35 (2016)

41. Lee, P. W., Severin, M. E. \& Lovett-Racke, A. E. TGF- $\beta$ regulation of T cells in multiple sclerosis. Eur. J. Immunol. 47, 446-453 (2017).

42. Bagur, M. J. et al. Influence of diet in multiple sclerosis: a systematic review. Adv. Nutr. 8, 463-472 (2017). 


\title{
Acknowledgements
}

We are grateful to the Italian Society of General Medicine (SIMG) for its support, and in particular to Anna Salvo MD, Guglielmo Travaglianti MD, Gaetano Profeta MD, Carmelo Di Gregorio MD, Antonino Rizzo MD, Nuccia Spada MD, Giovanni Cappello MD, Salvatore Amato MD, Marco Ciancio MD, Melchiorre Fidelbo MD, Cettina Persano MD, Valeria Polizzi MD, Giovanni Marotta MD, Maurizio D’Urso MD for their participation in the study.

\section{Author contributions}

A.N. designed the study and the research aims, conducted the analysis and wrote the manuscript. C.E.C. gathered participants data, conducted the analysis and wrote the manuscript. L.G. gathered participants' data and reviewed the manuscript. V.T., S.L., C.C., E.D., V.P. gathered participants' data. A.M. conducted the assay analysis. A.B., V.S., F.P., M.Z. reviewed the manuscript.

\section{Funding}

This research was funded by the Department of Medical and Surgical Sciences and Advanced Technologies "G.F. Ingrassia", University of Catania, Italy (Piano Triennale di Sviluppo delle Attività di Ricerca Scientifica del Dipartimento 2016-18).

\section{Competing interests}

The authors declare no competing interests.

\section{Additional information}

Supplementary information is available for this paper at https://doi.org/10.1038/s41598-020-75830-y.

Correspondence and requests for materials should be addressed to A.N.

Reprints and permissions information is available at www.nature.com/reprints.

Publisher's note Springer Nature remains neutral with regard to jurisdictional claims in published maps and institutional affiliations.

\begin{abstract}
Open Access This article is licensed under a Creative Commons Attribution 4.0 International License, which permits use, sharing, adaptation, distribution and reproduction in any medium or format, as long as you give appropriate credit to the original author(s) and the source, provide a link to the Creative Commons licence, and indicate if changes were made. The images or other third party material in this article are included in the article's Creative Commons licence, unless indicated otherwise in a credit line to the material. If material is not included in the article's Creative Commons licence and your intended use is not permitted by statutory regulation or exceeds the permitted use, you will need to obtain permission directly from the copyright holder. To view a copy of this licence, visit http://creativecommons.org/licenses/by/4.0/.
\end{abstract}

(C) The Author(s) 2020 